\title{
Molecule 3
}

\section{Singlet}

\section{UHF/STO-3G}

1 | 1 | UNPC-UNK |FOpt | UHF |STO-3G |C11H9N1 |PCUSER | 18-Oct-2002|0||\#UHF STO-3G OPT GUESS $=($ ALWAYS, MIX) || molecule 7|| $0,1 \mid C, 1.5190246997,0 ., 0.579798236$ $6|\mathrm{C}, 1.5130749495,0 ., 1.9972648708| \mathrm{H}, 2.465347361,0 ., 2.5148212848 \mid \mathrm{C}, 0.305$ $8933798,0 ., 2.7592901194|\mathrm{H}, 0.3640187854,0 ., 3.8401679138| \mathrm{C},-0.902816084$, $0 ., 2.1207829304|\mathrm{H},-1.8343977817,0 ., 2.6722364755| \mathrm{C},-0.9809820852,0 ., 0.6$ $178802668|\mathrm{C},-2.2775360915,0 ., 0.0209985637| \mathrm{H},-3.1510053598,0 ., 0.6599568$ $492|\mathrm{C},-2.4159271657,0 .,-1.3756949167| \mathrm{H},-3.3991067888,0 .,-1.8284673549 \mid$ $\mathrm{C},-1.2657959477,0 .,-2.1785485489|\mathrm{H},-1.3626831351,0 .,-3.2564964547| \mathrm{C}, 0$. $0582100826,0 .,-1.6161436821|\mathrm{C}, 1.1531766695,0 .,-2.5255239531| \mathrm{H}, 0.953251$ $1644,0 .,-3.5878689833|\mathrm{H}, 2.1772311078,0 .,-2.1929748939| \mathrm{C}, 0.1780653355,0$ $.,-0.159013584|\mathrm{~N}, 2.7154501823,0 .,-0.1247517178| \mathrm{H}, 3.4728669162,0 ., 0.605$ 3453704|| Version=x86-Win32-G98RevA.11.4|State=1-A' $|\mathrm{HF}=-470.3808365| \mathrm{S} 2=$ $2.379957|\mathrm{~S} 2-1=0.| \mathrm{S} 2 \mathrm{~A}=8.821057 \mid \mathrm{RMSD}=2$.997e-009 $|\mathrm{RMSF}=7.498 \mathrm{e}-005| \mathrm{Dipole}=-$ $0.1752937,0 ., 0.7037035|\mathrm{PG}=\mathrm{CS} \quad[\mathrm{SG}(\mathrm{C} 11 \mathrm{H} 9 \mathrm{~N} 1)]| \mid \mathrm{Q}$

\section{UCCSD(T)/STO-3G}

$1|1|$ UNPC-UNK $\mid$ SP | UCCSD (T)-FC $\mid$ STO-3G | C11H9N1 | PCUSER $18-0 C t-2002|0| \mid$ \#UCCS D-T/STO-3G GUESS=(ALWAYS, MIX) || molecule 7|| $0,1|\mathrm{C}| \mathrm{C}, 1,1.41747912 \mid \mathrm{H}, 2,1$. $08382996,1,118.28341605|\mathrm{C}, 2,1.4275748,1,122.50238876,3,180 ., 0| \mathrm{H}, 4,1.08$ $243955,2,119.18371448,1,180 ., 0|\mathrm{C}, 4,1.36699305,2,119.89261445,1,0 ., 0| \mathrm{H}$, $6,1.0825643,4,121.53092571,2,180 ., 0 \mid C, 6,1.504934,4,120.82276391,2,0 ., 0$ $|\mathrm{C}, 8,1.42734721,6,117.6967279,4,180 ., 0| \mathrm{H}, 9,1.08222745,8,119.0944111,6$, $0 ., 0|C, 9,1.40353296,8,120.37812546,6,180 ., 0| \mathrm{H}, 11,1.08242554,9,120.3856$ $1624,8,180 ., 0|\mathrm{C}, 11,1.40263173,9,119.25844927,8,0 ., 0| \mathrm{H}, 13,1.08229331,11$ $, 119.78110992,9,180 ., 0|C, 13,1.43850311,11,122.06830162,9,0 ., 0| C, 15,1.4$ $2334968,13,117.27546338,11,180 ., 0 \mid \mathrm{H}, 16,1.0809936,15,119.05196978,13,0$. , $0|\mathrm{H}, 16,1.07669697,15,122.29947634,13,180 ., 0| \mathrm{C}, 15,1.4620511,13,117.716$ $81937,11,0 ., 0|\mathrm{~N}, 1,1.38846123,19,120.65415542,8,180.0| \mathrm{H}, 20,1.05200849$, $1,105.55923301,19,180 ., 0||$ Version=x86-Win32-G98RevA.11.4|State=1-A' |HF $=-470.3808365|\mathrm{MP} 2=-470.9225035| \mathrm{MP} 3=-471.0199467|\mathrm{MP} 4 \mathrm{D}=-471.0526751| \mathrm{MP} 4 \mathrm{D}$ $\mathrm{Q}=-471.0462344|\mathrm{PUHF}=-469.726073| \mathrm{PMP} 2-0=-470.2624258 \mid \mathrm{PMP} 3-0=-470.351242$ $5|\mathrm{MP} 4 \mathrm{SDQ}=-471.0606849| \mathrm{CCSD}=-471.1340038|\mathrm{CCSD}(\mathrm{T})=-471.1536202| \mathrm{S} 2=2.3799$ $57|S 2-1=2.054427| \mathrm{S} 2 \mathrm{~A}=8.821057|\mathrm{RMSD}=2.997 \mathrm{e}-009| \mathrm{PG}=\mathrm{CS} \quad[\mathrm{SG}(\mathrm{C} 11 \mathrm{H} 9 \mathrm{~N} 1)]$ ] | @

\section{UB3LYP/STO-3G}

1 | 1 | UNPC-UNK | SP | UB3LYP |STO-3G |C11H 9N1 | PCUSER | 30-Jan-2003|0|| \#UB3LYP / ST O-3G GUESS $=($ ALWAYS, MIX) || molecule 7|| $0,1|\mathrm{C}| \mathrm{C}, 1,1.41747912 \mid \mathrm{H}, 2,1.083829$ $96,1,118.28341605|\mathrm{C}, 2,1.4275748,1,122.50238876,3,180 ., 0| \mathrm{H}, 4,1.08243955$ ,2,119.18371448,1,180., 0|C, 4, 1.36699305,2,119.89261445,1,0., 0| H, 6, 1.08 $25643,4,121.53092571,2,180 ., 0|C, 6,1.504934,4,120.82276391,2,0 ., 0| C, 8,1$ $.42734721,6,117.6967279,4,180 ., 0|\mathrm{H}, 9,1.08222745,8,119.0944111,6,0 \ldots, 0| \mathrm{C}$ $, 9,1.40353296,8,120.37812546,6,180 ., 0 \mid \mathrm{H}, 11,1.08242554,9,120.38561624,8$ $, 180 ., 0|\mathrm{C}, 11,1.40263173,9,119.25844927,8,0 ., 0| \mathrm{H}, 13,1.08229331,11,119.7$ 
$8110992,9,180 ., 0|C, 13,1.43850311,11,122.06830162,9,0 ., 0| C, 15,1.4233496$ $8,13,117.27546338,11,180 ., 0|\mathrm{H}, 16,1.0809936,15,119.05196978,13,0 ., 0| \mathrm{H}, 1$ $6,1.07669697,15,122.29947634,13,180 ., 0 \mid C, 15,1.4620511,13,117.71681937$, $11,0 ., 0|\mathrm{~N}, 1,1.38846123,19,120.65415542,8,180 ., 0| \mathrm{H}, 20,1.05200849,1,105$. $55923301,19,180,0||$ Version=x86-Win32-G98RevA.11.4| State=1-A' |HF=-473. $3239269|\mathrm{~S} 2=1.064986| \mathrm{S} 2-1=0 .|\mathrm{S} 2 \mathrm{~A}=0.590061| \mathrm{RMSD}=7.006 \mathrm{e}-005 \mid \mathrm{Dipole}=-0.274$ $8799,0.0 .6158344|\mathrm{PG}=\mathrm{CS} \quad[\mathrm{SG}(\mathrm{C} 11 \mathrm{H} 9 \mathrm{N1})]| \mid @$

\section{UHF/4-31G}

1 | 1 | UNPC-UNK | FOpt |UHF | 4-31G |C11H9N1 |PCUSER | 31-Jan-2003|0|| \#UHF/4-31G O $\mathrm{PT}$ GUESS $=($ ALWAYS, MIX) || molecule 7||0,1|C, 1.4903873429,0.,0.5645734277| $\mathrm{C}, 1.4881916337,0 ., 1.9734522232|\mathrm{H}, 2.435737038,0 ., 2.4782802518| \mathrm{C}, 0.30748$ $99307,0 ., 2.7223594806|\mathrm{H}, 0.352506445,0 ., 3.7928016856| \mathrm{C},-0.8901918215,0$. ,2.0729796497|H,-1.8129799114,0 . 2.6179826285|C, -0.9525062073,0.,0.622 $5352483|\mathrm{C},-2.2480608265,0 ., 0.0265194262| \mathrm{H},-3.1099368885,0 ., 0.663927418$ $1|\mathrm{C},-2.3801688495,0 .,-1.3382665579| \mathrm{H},-3.3514852182,0 .,-1.7915950898 \mid \mathrm{C}$, $-1.2411367197,0 .,-2.1353054047|\mathrm{H},-1.3460588908,0 .,-3.20228888| \mathrm{C}, 0.0641$ $285809,0 .,-1.6002802888|\mathrm{C}, 1.1328185267,0 .,-2.5195975231| \mathrm{H}, 0.9016591398$ $, 0 .,-3.5676361708|\mathrm{H}, 2.1512692371,0 \ldots,-2.2188468475| \mathrm{C}, 0.1949085103,0 .,-0$ $.146638933 \mid \mathrm{N}, 2.6477456599,0$., -0.1047649404|H, 3.4499088256,0 .,0.5067450 979 ||Version=x86-Win32-G98RevA.11.4|State=1-A' | HF=-475.5125203|S2=1.60 $2743|\mathrm{~S} 2-1=0.| \mathrm{S} 2 \mathrm{~A}=4.614714|\mathrm{RMSD}=7.782 \mathrm{e}-009| \mathrm{RMSF}=1.136 \mathrm{e}-004 \mid \mathrm{Dipole}=-0.28$ $57529,0.0 .9200565|\mathrm{PG}=\mathrm{CS} \quad[\mathrm{SG}(\mathrm{C} 11 \mathrm{H} 9 \mathrm{~N} 1)]| \mid \Theta$

\section{UCCSD(T)/4-31G}

1 | 1 | UNPC-UNK | SP | UCCSD ( T) -RW | 4-31G |C11H9N1 |PCUSER |01-Apr-2003|0|| \#UCCSD $(\mathrm{T}, \mathrm{WINDOW}=(13,110)) / 4-31 \mathrm{G}$ GUESS $=($ ALWAYS, $\mathrm{MIX})||$ molecule 7|| $0,1|\mathrm{C}| \mathrm{C}, 1,1$. $40888051|\mathrm{H}, 2,1.07363571,1,117.95825876| \mathrm{C}, 2,1.39818403,1,122.475841,3,1$ $80 ., 0|\mathrm{H}, 4,1.07138835,2,119.97844162,1,180 ., 0| \mathrm{C}, 4,1.36240073,2,119.1470$ $229,1,0 ., 0|\mathrm{H}, 6,1.07171176,4,120.96727136,2,180 ., 0| \mathrm{C}, 6,1.45178237,4,120$ $.92647436,2,0 ., 0|\mathrm{C}, 8,1.42607736,6,117.16468974,4,180 ., 0| \mathrm{H}, 9,1.07196982$ , 8, 118.81025234,6,0. $0|\mathrm{C}, 9,1.37116495,8,120.23351515,6,180 ., 0| \mathrm{H}, 11,1.0$ $7189656,9,120.54806661,8,180 ., 0|\mathrm{C}, 11,1.39020326,9,119.45357387,8,0 ., 0|$ $\mathrm{H}, 13,1.07212984,11,119.36629992,9,180 ., 0 \mid \mathrm{C}, 13,1.41066274,11,122.728950$ $42,9,0 ., 0|\mathrm{C}, 15,1.40969585,13,117.00834731,11,180 ., 0| \mathrm{H}, 16,1.07322862,15$ ,118.26481946,13,0., 0| H, 16,1.06192882,15,122.84497017,13,180.,0|C, 15, 1 $.45951245,13,117.42950221,11,0 ., 0 \mid \mathrm{N}, 1,1.33697125,19,121.19119485,8,180$ . , $0|\mathrm{H}, 20,1.00866757,1,112.63856855,19,180 ., 0| \mid$ Version=x86-Win32-G98ReV A. $11.4 \mid$ State $=1-A^{\prime}|\mathrm{HF}=-475.5125202| \mathrm{MP} 2=-476.376085|\mathrm{MP} 3=-476.4293382| \mathrm{MP} 4$ $\mathrm{D}=-476.4551262|\mathrm{MP} 4 \mathrm{DQ}=-476.4429336| \mathrm{PUHF}=-475.8286457 \mid \mathrm{PMP} 2-0=-476.688945$ $9|\mathrm{PMP} 3-0=-476.7371721| \mathrm{MP} 4 \mathrm{SDQ}=-476.458218|\mathrm{CCSD}=-476.4831405| \mathrm{CCSD}(\mathrm{T})=-47$ $6.5210574|\mathrm{~S} 2=1.602746| \mathrm{S} 2-1=1.414962|\mathrm{~S} 2 \mathrm{~A}=4.61473| \mathrm{RMSD}=6.164 \mathrm{e}-009 \mid \mathrm{PG}=\mathrm{CS}$ [SG (C11H9N1) ] |

\section{UB3LYP/4-31G}

1 | 1 | UNPC-UNK | SP | UB3LYP | 4-31G | C11H9N1 | PCUSER | 31-Jan-2003 |0|| \#UB3LYP / 4-3 1G GUESS = (ALWAYS, MIX) || molecule 7||0,1|C|C, 1, 1.40888051|H, 2, 1.07363571 , 1, 117.95825876|C,2,1.39818403,1,122.475841,3,180. , 0|H,4,1.07138835,2, $119.97844162,1,180 ., 0|\mathrm{C}, 4,1.36240073,2,119.1470229,1,0 ., 0| \mathrm{H}, 6,1.071711$ $76,4,120.96727136,2,180 ., 0|C, 6,1.45178237,4,120.92647436,2,0 ., 0| C, 8,1$. 
$42607736,6,117.16468974,4,180 ., 0|\mathrm{H}, 9,1.07196982,8,118.81025234,6,0 ., 0|$ $\mathrm{C}, 9,1.37116495,8,120.23351515,6,180 ., 0 \mid \mathrm{H}, 11,1.07189656,9,120.54806661$, $8,180 ., 0|\mathrm{C}, 11,1.39020326,9,119.45357387,8,0 ., 0| \mathrm{H}, 13,1.07212984,11,119$. $36629992,9,180 ., 0|\mathrm{C}, 13,1.41066274,11,122.72895042,9,0 ., 0| \mathrm{C}, 15,1.409695$ $85,13,117.00834731,11,180 ., 0|\mathrm{H}, 16,1.07322862,15,118.26481946,13,0 ., 0| \mathrm{H}$ $, 16,1.06192882,15,122.84497017,13,180 ., 0 \mid \mathrm{C}, 15,1.45951245,13,117.429502$ $21,11,0 ., 0|\mathrm{~N}, 1,1.33697125,19,121.19119485,8,180.0| \mathrm{H}, 20,1.00866757,1,1$ $12.63856855,19,180 ., 0||$ Version=x86-Win32-G98RevA.11.4 State=1-A' $\mid \mathrm{HF}=-4$ $78.6318343|\mathrm{~S} 2=0.959544| \mathrm{S} 2-1=0 .|\mathrm{S} 2 \mathrm{~A}=0.287885| \mathrm{RMSD}=2.110 \mathrm{e}-005 \mid \mathrm{Dipole}=-0$. $5968095,0.0 .7546176|\mathrm{PG}=\mathrm{CS} \quad[\mathrm{SG}(\mathrm{C} 11 \mathrm{H} 9 \mathrm{N1})]| \mid \mathrm{a}$

\section{UHF/6-311G(d)}

$1|1|$ UNPC-UNK |FOpt |UHF |6-311G (d) |C11H9N1 | PCUSER | 16-Dec-2003|0||\#P UHF/6 $-311 \mathrm{G}(\mathrm{D}) \quad \mathrm{OPT}$ SCF (CONVER=5) GEOM=CHECKPOINT GUESS=READ || mol 4|| $0,1 \mid \mathrm{C},-$ $1.2957168098,0.8762171826,0 .|\mathrm{C},-2.415302795,0.0674326362,0.| \mathrm{H},-3.38416$ $29955,0.5365070261,0 .|\mathrm{C},-2.3368458511,-1.3280140454,0.| \mathrm{H},-3.246023734$, $-1.9026788136,0 .|\mathrm{C},-1.1283191569,-1.9483722164,0.| \mathrm{H},-1.049050912,-3.02$ $0027435,0 .|C, 0.0457773635,-1.1618223593,0| C, 1.3047466197,$. $1,0 .|\mathrm{H}, 1.3042262361,-2.8991194331,0.| \mathrm{C}, 2.4846612834,-1.1507834972,0 . \mid \mathrm{H}$ ,3.4220956102,-1.6755215093,0.|C, $2.4506470491,0.2492681631,0$. | H, 3.3782 $349603,0.7916163075,0 .|C, 1.2674897552,0.9723699787,0| C, 1.3517614531,$. $.4077168218,0 .|\mathrm{H}, 2.407342926,2.677852348,0.| \mathrm{H},-0.6664575799,2.82885961$ $11,0 .|\mathrm{C}, 0.0028312081,0.2512359887,0.| \mathrm{N},-1.4553484476,2.2266268966,0 . \mid \mathrm{H}$ $,-2.3691460943,2.6041297318,0$. ||Version=x86-Win32-G98RevA.11.4 | State=1 $-\mathrm{A}^{\prime}|\mathrm{HF}=-476.2123887| \mathrm{S} 2=0 .|\mathrm{S} 2-1=0.| \mathrm{S} 2 \mathrm{~A}=0 .|\mathrm{RMSD}=3.829 \mathrm{e}-006| \mathrm{RMSF}=4.762 \mathrm{e}-0$ $05 \mid$ Dipole $=0.4159024,-0.718673,0 .|\mathrm{PG}=\mathrm{CS} \quad[\mathrm{SG}(\mathrm{C} 11 \mathrm{H} 9 \mathrm{~N} 1)]| \mid \mathrm{e}$

\section{UB3LYP/6-311G(d)}

$1|1|$ UNPC-UNK $\mid$ SP |UB3LYP |6-311G (d) |C11H9N1 |PCUSER | 16-DeC-2003|0|| \#P UB3L YP/6-311G (D) SCF (CONVER=5) GEOM=CHECKPOINT GUESS=READ || $\operatorname{mol} 4|| 0,1 \mid \mathrm{C}$, $1.2957168098,0.8762171826,0 .|\mathrm{C},-2.415302795,0.0674326362,0.| \mathrm{H},-3.38416$ $29955,0.5365070261,0 .|\mathrm{C},-2.3368458511,-1.3280140454,0.| \mathrm{H},-3.246023734$, $-1.9026788136,0 .|\mathrm{C},-1.1283191569,-1.9483722164,0.| \mathrm{H},-1.049050912,-3.02$ $0027435,0 .|C, 0.0457773635,-1.1618223593,0| C, 1.3047466197,$. $1,0 .|\mathrm{H}, 1.3042262361,-2.8991194331,0.| \mathrm{C}, 2.4846612834,-1.1507834972,0 . \mid \mathrm{H}$ $, 3.4220956102,-1.6755215093,0 .|\mathrm{C}, 2.4506470491,0.2492681631,0.| \mathrm{H}, 3.3782$ $349603,0.7916163075,0 .|C, 1.2674897552,0.9723699787,0| C, 1.3517614531,$. $.4077168218,0 . \mid \mathrm{H}, 2.407342926,2.677852348,0$. | $\mathrm{H},-0.6664575799,2.82885961$ $11,0 .|\mathrm{C}, 0.0028312081,0.2512359887,0.| \mathrm{N},-1.4553484476,2.2266268966,0 . \mid \mathrm{H}$ $,-2.3691460943,2.6041297318,0$. ||Version=x86-Win32-G98RevA.11.4 | State=1 $-\mathrm{A}^{\prime}|\mathrm{HF}=-479.3249451| \mathrm{S} 2=0 .|\mathrm{S} 2-1=0.| \mathrm{S} 2 \mathrm{~A}=0 .|\mathrm{RMSD}=4.781 \mathrm{e}-006| \mathrm{Dipole}=0.0877$ $187,-0.9042944,0 .|P G=C S[S G(C 11 H 9 N 1)]| \mid$ e

\section{RHF/STO-3G}

1 | 1 UNPC-UNK |FOpt |ROHF |STO-3G|C11H9N1 |PCUSER|14-DeC-2003|0||\#P ROHF/ST O-3G OPT SCF (CONVER=5)||molecule 4||0,1|C,1.4676071699,0.,0.531168362 $4|\mathrm{C}, 1.5096856703,0 ., 1.9048136889| \mathrm{H}, 2.4713278601,0 ., 2.3962719645 \mid \mathrm{C}, 0.33$ $28184235,0 ., 2.6803419681|\mathrm{H}, 0.4172454111,0 ., 3.7604286451| \mathrm{C},-0.883614249$ $2,0 ., 2.0850630872|\mathrm{H},-1.7946886558,0 ., 2.6653116387| \mathrm{C},-0.9776305027,0 ., 0$ $.6619792865|\mathrm{C},-2.2684335463,0 ., 0.0395523739| \mathrm{H},-3.1415739113,0 ., 0.68267$ $88607|\mathrm{C},-2.4112525516,0 .,-1.3077721355| \mathrm{H},-3.3904803075,0 .,-1.766899140$ 
$3|\mathrm{C},-1.2527797016,0 .,-2.119007843| \mathrm{H},-1.3758210254,0 .,-3.1972406418 \mid \mathrm{C}, 0$ $.0186331619,0 .,-1.5916406384|\mathrm{C}, 1.1869348819,0 .,-2.5094915232| \mathrm{H}, 0.75471$ $40918,0 .,-3.5362469916|\mathrm{H}, 2.5801497974,0 .,-1.2234324449| \mathrm{C}, 0.1793715657$, $0 .,-0.1448569592|\mathrm{~N}, 2.647822882,0 .,-0.2058904206| \mathrm{H}, 3.5363246349,0 ., 0.27$ 94630468|| Version=x86-Win32-G98RevA.11.4 |State $=1-A^{\prime}|\mathrm{HF}=-470.2585031| \mathrm{RM}$ $\mathrm{SD}=1.999 \mathrm{e}-006|\mathrm{RMSF}=4.996 \mathrm{e}-005| \mathrm{Dipole}=-0.5529849,0 .,-0.2870239 \mid \mathrm{PG}=\mathrm{CS}[\mathrm{S}$ $\mathrm{G}(\mathrm{C} 11 \mathrm{H} 9 \mathrm{~N} 1)]||$ e

\section{RHF/6-311G(d)}

1 | 1 | UNPC-UNK |FOpt |ROHF |6-311G (d) |C11H9N1 | PCUSER | 14-DeC-2003|0|| \#P ROHF /6-311G (D) OPT SCF (CONVER=5) GEOM=CHECKPOINT GUESS=READ||molecule 4|| $0,1|\mathrm{C}, 1.4775198909,0 ., 0.5132903639| \mathrm{C}, 1.4996551044,0 ., 1.894456016 \mid \mathrm{H}, 2.4$ $554042385,0.2 .3897738635|\mathrm{C}, 0.3349694553,0 ., 2.6668339335| \mathrm{H}, 0.418763875$ $5,0 ., 3.7391358949|\mathrm{C},-0.8854947039,0 ., 2.0701046718| \mathrm{H},-1.7913665433,0 ., 2$ $.6481537866|C,-0.9581570054,0 ., 0.6589209071| C,-2.2414023278,0 ., 0.04608$ $72673|\mathrm{H},-3.1030580814,0 ., 0.6903575521| \mathrm{C},-2.4091743473,0 .,-1.3017067464$ $|\mathrm{H},-3.3908741913,0 .,-1.7380479024| \mathrm{C},-1.2675249874,0 .,-2.1126135027 \mid \mathrm{H},-$ $1.387804595,0 .,-3.1803942223|C, 0.0199287879,0 .,-1.5974456027| C, 1.12012$ $50121,0 .,-2.5222956417|\mathrm{H}, 0.7085167614,0 .,-3.5312297654| \mathrm{H}, 2.6633139853$, $0 .,-1.160225703|\mathrm{C}, 0.1995724067,0 .,-0.1526865088| \mathrm{N}, 2.6546358431,0 \ldots,-0.1$ $67553575|\mathrm{H}, 3.5045499352,0 ., 0.3376805767| \mid$ Version=x86-Win32-G98RevA.11. $4 \mid$ State $=1-A^{\prime}|H F=-476.212388| R M S D=6.153 e-006|R M S F=4.365 e-005| D i p o l e=-0$. $8242628,0 ., 0.0955398|\mathrm{PG}=\mathrm{CS} \quad[\mathrm{SG}(\mathrm{C} 11 \mathrm{H} 9 \mathrm{~N} 1)]| \mid \Theta$

\section{RHF/6-311G(d,p)}

1 | 1 | UNPC-UNK |FOpt |ROHF |6-311G (d,p) |C11H9N1 | PCUSER | 15-DeC-2003|0|| \#P RO $\mathrm{HF} / 6-311 * *$ OPT SCF (CONVER=5) GEOM=CHECKPOINT GUESS=READ||molecule 4|| $0,1|\mathrm{C}, 1.4773627041,0 ., 0.5138718602| \mathrm{C}, 1.49999185,0 ., 1.8946583849 \mid \mathrm{H}, 2.45$ $6037027,0 ., 2.3890342489|\mathrm{C}, 0.3347679049,0 ., 2.6668566421| \mathrm{H}, 0.4187022624$, $0 ., 3.739233399|\mathrm{C},-0.8855723605,0 ., 2.0705634961| \mathrm{H},-1.7919918664,0 ., 2.64$ $74928976|C,-0.9584667506,0 ., 0.6590018317| C,-2.2413864235,0 ., 0.04573036$ $98|\mathrm{H},-3.1028383806,0 ., 0.6906724529| \mathrm{C},-2.4081386015,0 .,-1.3021935607 \mid \mathrm{H}$, $-3.389485041,0 .,-1.7393676566|\mathrm{C},-1.2661469551,0 .,-2.1124801544| \mathrm{H},-1.38$ $41028562,0 .,-3.1808133142|\mathrm{C}, 0.0207272044,0 .,-1.5961605256| \mathrm{C}, 1.12188972$ $04,0 .,-2.5201622814|\mathrm{H}, 0.7108040247,0 .,-3.5298696358| \mathrm{H}, 2.6500803061,0 .$, $-1.1665471536|\mathrm{C}, 0.1991417563,0 .,-0.1518289194| \mathrm{N}, 2.6522271761,0 .,-0.170$ $5100621|\mathrm{H}, 3.5021839979,0 ., 0.3365923361| \mid$ Version=x86-Win32-G98RevA.11.4 $\mid$ St ate $=1-A^{\prime}|\mathrm{HF}=-476.2320665| \mathrm{RMSD}=4.044 \mathrm{e}-006|\mathrm{RMSF}=3.804 \mathrm{e}-005| \mathrm{Dipole}=-0$. $8334434,0 ., 0.0808256|\mathrm{PG}=\mathrm{CS} \quad[\mathrm{SG}(\mathrm{C} 11 \mathrm{H} 9 \mathrm{~N} 1)]| \mid @$

\section{RB3LYP/6-311G(d,p)}

1 | 1 | UNPC-UNK | FOpt |ROB3LYP | 6-311G (d, p) |C11H9N1 | PCUSER | 15-Dec-2003|0|| \#P ROB3LYP/6-311** OPT SCF (CONVER=5) GEOM=CHECKPOINT GUESS=READ||molecu le 4|| $0,1|C,-1.2942774894,0.8826886404,0| C,-2.4324547017,$. , $0 .|\mathrm{H},-3.4074072833,0.5390221725,0.| \mathrm{C},-2.3540954843,-1.3345354606,0$. | H $,-3.271140915,-1.913342313,0 .|\mathrm{C},-1.1330238583,-1.9674675786,0.| \mathrm{H},-1.05$ $5519747,-3.0479064597,0 . \mid C, 0.048813577,-1.1833417277,0$. |C, 1.3073203675 $,-1.8302470425,0 .|\mathrm{H}, 1.3176375402,-2.9159134016,0.| \mathrm{C}, 2.5022236025,-1.13$ $53187464,0 .|\mathrm{H}, 3.4474118987,-1.6653747765,0.| \mathrm{C}, 2.4659535885,0.256517029$ 
$8,0 .|\mathrm{H}, 3.392449608,0.8204579789,0.| \mathrm{C}, 1.255995417,0.9883768407,0 . \mid \mathrm{C}, 1.2$ $922603997,2.4130570789,0 .|\mathrm{H}, 2.3487674714,2.7296689286,0.| \mathrm{H},-0.52929843$ $83,2.7775850753,0 .|\mathrm{C}, 0.0011370306,0.248591664,0.| \mathrm{N},-1.4109137557,2.232$ $210915,0 . \mid \mathrm{H},-2.3256185394,2.6505750074,0$. | |Version=x86-Win32-G98RevA. 1 $1.4 \mid$ State $=1-A^{\prime}|\mathrm{HF}=-479.3480641| \mathrm{RMSD}=3.149 \mathrm{e}-006|\mathrm{RMSF}=8.378 \mathrm{e}-005| \mathrm{Dipole}=$ $0.1456251,-0.916475,0 .|P G=C S[S G(C 11 \mathrm{H} 9 \mathrm{~N} 1)]| \mid \Theta$

\section{Triplet}

\section{UHF/STO-3G}

$1|1|$ UNPC-UNK |FOpt | UHF |STO-3G |C11H9N1 (3) |PCUSER | 18-OCt-2002|0||\#UHF STO -3G OPT GUESS= (ALWAYS, MIX) || molecule 7|| $0,3 \mid C, 1.4882463613,0 ., 0.587599$ $9706|\mathrm{C}, 1.4756962828,0 ., 2.0301199445| \mathrm{H}, 2.4270531971,0 ., 2.5484736152 \mid \mathrm{C}, 0$ $.2739494021,0 ., 2.7638904734|\mathrm{H}, 0.2981911049,0 ., 3.8460910501| \mathrm{C},-0.955594$ $7012,0 ., 2.0763829765|\mathrm{H},-1.886193359,0 ., 2.6290752234| \mathrm{C},-1.0073817199,0$. $, 0.6404802271|\mathrm{C},-2.2897712053,0 .,-0.0063975258| \mathrm{H},-3.1808983379,0 ., 0.60$ $76808994|\mathrm{C},-2.3923230568,0 .,-1.4112814174| \mathrm{H},-3.3647769304,0 .,-1.886632$ $5753|\mathrm{C},-1.2225717119,0 .,-2.1942269811| \mathrm{H},-1.3017743014,0 .,-3.2734793515$ |C, $0.1015527655,0 .,-1.6133795135|\mathrm{C}, 1.1975392582,0 .,-2.5143118114| \mathrm{H}, 1.0$ $025954941,0 .,-3.5777991504|\mathrm{H}, 2.220858219,0 .,-2.1806572238| \mathrm{C}, 0.21642636$ $35,0 .,-0.1470555359|\mathrm{~N}, 2.7168179192,0 .,-0.0920893385| \mathrm{H}, 3.45261125,0 ., 0$. 6609480402|| Version=x86-Win32-G98RevA.11.4|State=3-A'|HF=-470.437518|S $2=3.797684|\mathrm{~S} 2-1=0.| \mathrm{S} 2 \mathrm{~A}=5.356412|\mathrm{RMSD}=1.435 \mathrm{e}-009| \mathrm{RMSF}=1.025 \mathrm{e}-004 \mid \mathrm{Dipole}$ $=-0.144855,0 ., 0.6523695|\mathrm{PG}=\mathrm{CS} \quad[\mathrm{SG}(\mathrm{C} 11 \mathrm{H} 9 \mathrm{~N} 1)]| \mid \mathrm{e}$

\section{UCCSD(T)/STO-3G}

1 | 1 UNPC-UNK $\mid$ SP | UCCSD (T) -FC |STO-3G|C11H9N1 (3) |PCUSER | 18-Oct-2002|0||\#U CCSD-T/STO-3G GUESS $=($ ALWAYS, MIX) || molecule 7||0,3|C $|\mathrm{C}, 1,1.44257457| \mathrm{H}, 2$ $, 1.0834069,1,118.08560896|\mathrm{C}, 2,1.40805353,1,121.90614615,3,180 ., 0| \mathrm{H}, 4,1$ $.08247205,2,120.12444675,1,180 ., 0|\mathrm{C}, 4,1.4087034,2,119.3803017,1,0 ., 0| \mathrm{H}$ $, 6,1.08235049,4,120.08146295,2,180 ., 0 \mid C, 6,1.43683632,4,121.27754335,2$, $0 ., 0|\mathrm{C}, 8,1.43630555,6,118.83334386,4,180 ., 0| \mathrm{H}, 9,1.08221988,8,118.66133$ $523,6,0 ., 0|\mathrm{C}, 9,1.40862189,8,120.94281585,6,180 ., 0| \mathrm{H}, 11,1.0824164,9,120$ $.22512785,8,180 ., 0|\mathrm{C}, 11,1.40759439,9,119.62044714,8,0 ., 0| \mathrm{H}, 13,1.082154$ $67,11,119.5982289,9,180 ., 0|C, 13,1.44592165,11,122.51916223,9,0 ., 0| C, 15$ $, 1.41875488,13,116.89342139,11,180 ., 0 \mid \mathrm{H}, 16,1.08120691,15,119.03383365$, $13,0 ., 0|\mathrm{H}, 16,1.07633967,15,122.5201848,13,180 ., 0| \mathrm{C}, 15,1.47081676,13,11$ $8.16486604,11,0 ., 0|\mathrm{~N}, 1,1.40405329,19,121.03458122,8,180 ., 0| \mathrm{H}, 20,1.0528$ $329,1,105.38349146,19,180 ., 0||$ Version=x86-Win32-G98RevA.11.4 State=3-A ' $|\mathrm{HF}=-470.437518| \mathrm{MP} 2=-470.9411508|\mathrm{MP} 3=-471.0366991| \mathrm{MP} 4 \mathrm{D}=-471.0672798 \mid \mathrm{M}$ $\mathrm{P} 4 \mathrm{DQ}=-471.0623637|\mathrm{PUHF}=-470.5759199| \mathrm{PMP} 2-0=-471.0730622 \mid \mathrm{PMP} 3-0=-471.15$ $88565|\mathrm{MP} 4 \mathrm{SDQ}=-471.0754343| \mathrm{CCSD}=-471.1429358|\mathrm{CCSD}(\mathrm{T})=-471.1602153| \mathrm{S} 2=3$. $797684|\mathrm{~S} 2-1=3.412986| \mathrm{S} 2 \mathrm{~A}=5.356412|\mathrm{RMSD}=1.435 \mathrm{e}-009| \mathrm{PG}=\mathrm{CS} \quad[\mathrm{SG}(\mathrm{C} 11 \mathrm{H} 9 \mathrm{~N} 1)] \mid$ | @

\section{UB3LYP/STO-3G}

1 | 1 | UNPC-UNK | SP | UB3LYP |STO-3G |C11H9N1 (3) |PCUSER | 30-Jan-2003|0|| \#UB3LYP /STO-3G GUESS=(ALWAYS,MIX) || molecule 7||0,3|C|C, 1, 1.44257457| H, 2, 1.083 $4069,1,118.08560896|\mathrm{C}, 2,1.40805353,1,121.90614615,3,180 ., 0| \mathrm{H}, 4,1.08247$ $205,2,120.12444675,1,180 ., 0|\mathrm{C}, 4,1.4087034,2,119.3803017,1,0 ., 0| \mathrm{H}, 6,1.0$ $8235049,4,120.08146295,2,180 ., 0|C, 6,1.43683632,4,121.27754335,2,0 ., 0| C$ 
$, 8,1.43630555,6,118.83334386,4,180 ., 0 \mid \mathrm{H}, 9,1.08221988,8,118.66133523,6$, $0 ., 0|C, 9,1.40862189,8,120.94281585,6,180 ., 0| \mathrm{H}, 11,1.0824164,9,120.22512$ $785,8,180 ., 0|\mathrm{C}, 11,1.40759439,9,119.62044714,8,0 ., 0| \mathrm{H}, 13,1.08215467,11$, $119.5982289,9,180 ., 0|C, 13,1.44592165,11,122.51916223,9,0 ., 0| C, 15,1.418$ $75488,13,116.89342139,11,180.0 \mid \mathrm{H}, 16,1.08120691,15,119.03383365,13,0 .$, $0|\mathrm{H}, 16,1.07633967,15,122.5201848,13,180 ., 0| \mathrm{C}, 15,1.47081676,13,118.1648$ $6604,11,0 ., 0|\mathrm{~N}, 1,1.40405329,19,121.03458122,8,180 ., 0| \mathrm{H}, 20,1.0528329,1$, $105.38349146,19,180 ., 0||$ Version=x86-Win32-G98RevA.11.4| State=3-A' |HF=$473.336213|\mathrm{~S} 2=2.158545| \mathrm{S} 2-1=0 .|\mathrm{S} 2 \mathrm{~A}=2.014318| \mathrm{RMSD}=4.479 \mathrm{e}-005 \mid \mathrm{Dipole}=-0$. $2355193,-0.0000854,0.5388729|P G=C S \quad[S G(C 11 H 9 N 1)]| \mid a$

\section{UHF/4-31G}

1 | 1 |UNPC-UNK |FOpt |UHF | 4-31G |C11H9N1 (3) |PCUSER | 05-DeC-2002|0|| \#UHF 4-31 G OPT GUESS $=$ (ALWAYS, MIX) || molecule 7||0,3|C, 1.4818529211,0.,0.57541118 $65|\mathrm{C}, 1.4692469275,0 ., 2.0037993252| \mathrm{H}, 2.4142790004,0 ., 2.5133643208 \mid \mathrm{C}, 0.2$ $878011114,0 ., 2.7307951276|\mathrm{H}, 0.3128002227,0 ., 3.8020891101| \mathrm{C},-0.92747116$ $42,0 ., 2.054523|\mathrm{H},-1.8485963067,0 ., 2.6036236641| \mathrm{C},-0.9853769969,0 ., 0.63$ $57779442|\mathrm{C},-2.2569734744,0 ., 0.0081403019| \mathrm{H},-3.1348019415,0 ., 0.62439249$ $33|\mathrm{C},-2.3685320012,0 .,-1.3772316093| \mathrm{H},-3.3348048691,0 .,-1.841419876 \mid \mathrm{C}$, $-1.2209703002,0 .,-2.1578978712|\mathrm{H},-1.3076459945,0 .,-3.2266231157| \mathrm{C}, 0.09$ $67228763,0 .,-1.6009720577|\mathrm{C}, 1.162632476,0 .,-2.5120476462| \mathrm{H}, 0.938274770$ $5,0 .,-3.5616778668|\mathrm{H}, 2.1809722866,0 .,-2.2103981477| \mathrm{C}, 0.2209066984,0$, , $0.1497568733|\mathrm{~N}, 2.6536906323,0 .,-0.0741108772| \mathrm{H}, 3.4446539625,0 ., 0.55218$ $05924 \mid$ Version=x86-Win32-G98RevA.11.4|State=3-A' $|\mathrm{HF}=-475.550308| \mathrm{S} 2=3.2$ $05492|\mathrm{~S} 2-1=0.| \mathrm{S} 2 \mathrm{~A}=3.336164|\mathrm{RMSD}=4.336 \mathrm{e}-009| \mathrm{RMSF}=3.166 \mathrm{e}-005 \mid \mathrm{Dipole}=-0.1$ $746714,0 ., 0.8337444|\mathrm{PG}=\mathrm{CS} \quad[\mathrm{SG}(\mathrm{C} 11 \mathrm{H} 9 \mathrm{~N} 1)]| \mid \mathrm{e}$

\section{$\operatorname{UCCSD}(T) / 4-31 G$}

1 | 1 | UNPC-UNK | SP | UCCSD (T) -RW | 4-31G |C11H9N1 (3) | PCUSER |01-Apr-2003|0|| \#UC $\operatorname{CSD}(\mathrm{T}, \mathrm{WINDOW}=(13,110)) / 4-31 \mathrm{G}$ GUESS $=($ ALWAYS, MIX) || molecule 7|| $0,3|\mathrm{C}| \mathrm{C}, 1$ $, 1.42844376|\mathrm{H}, 2,1.07365828,1,117.82807915| \mathrm{C}, 2,1.38720478,1,122.1114625$ $6,3,180 ., 0|\mathrm{H}, 4,1.07158563,2,120.26904231,1,180 ., 0| \mathrm{C}, 4,1.39076623,2,119$ $.29920871,1,0 ., 0|\mathrm{H}, 6,1.07237263,4,120.10503608,2,180 ., 0| \mathrm{C}, 6,1.41992627$ $, 4,121.43218967,2,0 ., 0|\mathrm{C}, 8,1.41805734,6,118.60735567,4,180 ., 0| \mathrm{H}, 9,1.07$ $254351,8,118.66028319,6,0 ., 0|\mathrm{C}, 9,1.38985634,8,120.87400437,6,180 ., 0| \mathrm{H}$, $11,1.07198601,9,120.26305422,8,180 ., 0 \mid C, 11,1.38792567,9,119.62290415,8$ $, 0 ., 0|\mathrm{H}, 13,1.07223427,11,119.59012194,9,180 ., 0| \mathrm{C}, 13,1.43055292,11,122$. $86177409,9,0 ., 0|\mathrm{C}, 15,1.40222038,13,116.56671085,11,180 ., 0| \mathrm{H}, 16,1.07334$ $057,15,118.45646825,13,0 ., 0|\mathrm{H}, 16,1.06207739,15,122.97798262,13,180 ., 0|$ $\mathrm{C}, 15,1.45651884,13,117.80246957,11,0 ., 0 \mid \mathrm{N}, 1,1.3398069,19,121.09829283$, $8,180 ., 0|\mathrm{H}, 20,1.00889246,1,112.62894849,19,180 ., 0| \mid$ Version=x86-Win32-G 98RevA.11.4 $\mid$ State=3-A $|\mathrm{HF}=-475.550308| \mathrm{MP} 2=-476.3619673 \mid \mathrm{MP} 3=-476.424364$ $|\mathrm{MP} 4 \mathrm{D}=-476.4476554| \mathrm{MP} 4 \mathrm{DQ}=-476.4386469|\mathrm{PUHF}=-475.6245292| \mathrm{PMP} 2-0=-476.43$ $06423|\mathrm{PMP} 3-0=-476.4843582| \mathrm{MP} 4 \mathrm{SDQ}=-476.4556113|\mathrm{CCSD}=-476.4914891| \mathrm{CCSD}(\mathrm{T}$ )$=-476.5266623|\mathrm{~S} 2=3.205492| \mathrm{S} 2-1=2.906017|\mathrm{~S} 2 \mathrm{~A}=3.336164| \mathrm{RMSD}=6.536 \mathrm{e}-009 \mid$ $\mathrm{PG}=\mathrm{CS} \quad[\mathrm{SG}(\mathrm{C} 11 \mathrm{H} 9 \mathrm{~N} 1)]||$ @

\section{UB3LYP/4-31G}

1 | 1 | UNPC-UNK | SP | UB3LYP | 4-31G |C11H9N1 (3) | PCUSER | 31-Jan-2003 | 0 || \#UB3LYP / 
4-31G GUESS $=($ ALWAYS, MIX) || molecule 7||0,3|C|C, 1, 1.42844376|H, $2,1.07365$ $828,1,117.82807915|\mathrm{C}, 2,1.38720478,1,122.11146256,3,180 ., 0| \mathrm{H}, 4,1.071585$ $63,2,120.26904231,1,180 ., 0|\mathrm{C}, 4,1.39076623,2,119.29920871,1,0 ., 0| \mathrm{H}, 6,1$. $07237263,4,120.10503608,2,180 ., 0|C, 6,1.41992627,4,121.43218967,2,0 ., 0|$ $\mathrm{C}, 8,1.41805734,6,118.60735567,4,180.0 \mid \mathrm{H}, 9,1.07254351,8,118.66028319,6$ $, 0 ., 0|\mathrm{C}, 9,1.38985634,8,120.87400437,6,180 ., 0| \mathrm{H}, 11,1.07198601,9,120.263$ $05422,8,180 ., 0|\mathrm{C}, 11,1.38792567,9,119.62290415,8,0 ., 0| \mathrm{H}, 13,1.07223427,1$ $1,119.59012194,9,180 ., 0|C, 13,1.43055292,11,122.86177409,9,0 ., 0| C, 15,1$. $40222038,13,116.56671085,11,180.0 \mid \mathrm{H}, 16,1.07334057,15,118.45646825,13$, $0 ., 0|\mathrm{H}, 16,1.06207739,15,122.97798262,13,180 ., 0| \mathrm{C}, 15,1.45651884,13,117$. $80246957,11,0 ., 0|\mathrm{~N}, 1,1.3398069,19,121.09829283,8,180 ., 0| \mathrm{H}, 20,1.0088924$ $6,1,112.62894849,19,180 ., 0||$ Version=x86-Win32-G98RevA $.11 .4 \mid \mathrm{HF}=-478.637$ $906|\mathrm{~S} 2=2.096181| \mathrm{S} 2-1=0 .|\mathrm{S} 2 \mathrm{~A}=2.005165| \mathrm{RMSD}=6.505 e-005 \mid \mathrm{Dipole}=-0.3595235$ $, 0.0008265,0.7171236|\mathrm{PG}=\mathrm{CS}[\mathrm{SG}(\mathrm{C} 11 \mathrm{H} 9 \mathrm{~N} 1)]| \mid @$

\section{UHF/6-311G(d)}

1 | 1 | UNPC-UNK | FOpt | UHF |6-311G (d) |C11H9N1 (3) |PCUSER | 16-Dec-2003|0|| \#P UH F/6-311G (D) OPT SCF (CONVER=5) GUESS=READ GEOM=CHECKPOINT $\mid$ molecule $4 \mid$ $|0,3| \mathrm{C}, 1.4968188342,0 ., 0.5617253272 \mid \mathrm{C}, 1.4907935143,0$. 1.997138645|H, 2 . $4413259608,0.2 .5017171514|\mathrm{C}, 0.3142076556,0 ., 2.7319571257| \mathrm{H}, 0.34635782$ $88,0 ., 3.8063432067|\mathrm{C},-0.907330154,0 ., 2.0629899232| \mathrm{H},-1.8258878025,0 ., 2$ $.6214375541|\mathrm{C},-0.9761526281,0 ., 0.6423893671| \mathrm{C},-2.2565591711,0 ., 0.02846$ $11384|\mathrm{H},-3.1306274707,0 ., 0.6544708715| \mathrm{C},-2.3828561035,0 .,-1.3571685672$ $|\mathrm{H},-3.3565975626,0 .,-1.8129984573| \mathrm{C},-1.2429110802,0,-2.1496133736 \mid \mathrm{H},-$ $1.3435560845,0 \ldots,-3.2197977574|\mathrm{C}, 0.0825999213,0 .,-1.6062792719| \mathrm{C}, 1.1335$ $254784,0 .,-2.5339801176|\mathrm{H}, 0.8887812588,0 .,-3.5812947034| \mathrm{H}, 2.16148858,0$ $.,-2.257535657|\mathrm{C}, 0.2214147559,0 .,-0.1540071154| \mathrm{N}, 2.6523027295,0 .,-0.08$ $88117587|\mathrm{H}, 3.4112900485,0.0 .5676616162| \mid$ Version=x86-Win32-G98RevA.11.

$4\left|\mathrm{State}=3-\mathrm{A}^{\prime}\right| \mathrm{HF}=-476.3133111|\mathrm{~S} 2=3.135908| \mathrm{S} 2-1=0 .|\mathrm{S} 2 \mathrm{~A}=3.16137| \mathrm{RMSD}=8.14$ $3 e-006|\mathrm{RMSF}=9.042 \mathrm{e}-005| \mathrm{Dipole}=-0.2034838,0 ., 0.7462465 \mid \mathrm{PG}=\mathrm{CS} \quad$ [SG (C11H9N 1) $]||[$

\section{UB3LYP/6-311G(d)}

1 | 1 | UNPC-UNK | SP | UB3LYP |6-311G (d) |C11H9N1 (3) |PCUSER|16-DeC-2003|0||\#P U B3LYP/6-311G (D) SP SCF (CONVER=5) GUESS=READ GEOM=CHECKPOINT ||molecule 4|| $0,3|\mathrm{C}, 1.4968188342,0 ., 0.5617253272| \mathrm{C}, 1.4907935143,0 ., 1.997138645 \mid \mathrm{H}$ ,2.4413259608,0.,2.5017171514|C,0.3142076556,0.,2.7319571257|H,0.34635 $78288,0 ., 3.8063432067|\mathrm{C},-0.907330154,0 ., 2.0629899232| \mathrm{H},-1.8258878025,0$ $.2 .6214375541|\mathrm{C},-0.9761526281,0 ., 0.6423893671| \mathrm{C},-2.2565591711,0 ., 0.02$ $84611384|\mathrm{H},-3.1306274707,0 ., 0.6544708715| \mathrm{C},-2.3828561035,0 .,-1.3571685$ $672 \mid \mathrm{H},-3.3565975626,0$., $-1.8129984573|\mathrm{C},-1.2429110802,0 .,-2.1496133736|$ $\mathrm{H},-1.3435560845,0 .,-3.2197977574|\mathrm{C}, 0.0825999213,0 .,-1.6062792719| \mathrm{C}, 1.1$ $335254784,0 .,-2.5339801176|\mathrm{H}, 0.8887812588,0 .,-3.5812947034| \mathrm{H}, 2.1614885$ $8,0 .,-2.257535657|\mathrm{C}, 0.2214147559,0 .,-0.1540071154| \mathrm{N}, 2.6523027295,0 .,-0$ $.0888117587|\mathrm{H}, 3.4112900485,0 ., 0.5676616162| \mid$ Version=x86-Win32-G98RevA. $11.4 \mid$ State=3-A' $|\mathrm{HF}=-479.3636018| \mathrm{S} 2=2.093052|\mathrm{~S} 2-1=0.| \mathrm{S} 2 \mathrm{~A}=2.00491 \mid \mathrm{RMSD}=7$ $.437 e-006|\mathrm{Dipole}=-0.4050741,0 ., 0.6680714| \mathrm{PG}=\mathrm{CS} \quad[\mathrm{SG}(\mathrm{C} 11 \mathrm{H} 9 \mathrm{~N} 1)]||$ e

\section{ROHF/STO-3G}


1 1| UNPC-UNK |FOpt |ROHF |STO-3G|C11H9N1 (3)|PCUSER | 15-DeC-2003|0||\#P ROHF /STO-3G OPT SCF (CONVER=5)||molecule 4||0,3|C, 1.4566648459,0.,0.576268 $2577|\mathrm{C}, 1.4795653765,0 ., 1.941254587| \mathrm{H}, 2.4335817628,0 ., 2.4545353616 \mid \mathrm{C}, 0$. $290033861,0 ., 2.7102910607|\mathrm{H}, 0.353375819,0 ., 3.7905628138| \mathrm{C},-0.903154308$ $4,0 ., 2.0774038926|\mathrm{H},-1.8286306364,0 ., 2.6391663619| \mathrm{C},-0.9828020733,0 ., 0$ $.6481470974|\mathrm{C},-2.2710512906,0 ., 0.0284306021| \mathrm{H},-3.1461287441,0 ., 0.66464$ $16272|\mathrm{C},-2.3854907304,0 .,-1.3176431868| \mathrm{H},-3.3571781113,0 .,-1.794911242$ $7|\mathrm{C},-1.218474697,0 .,-2.117995353| \mathrm{H},-1.3297231906,0 .,-3.1941559463 \mid \mathrm{C}, 0$. $0433558859,0 .,-1.5837519094|\mathrm{C}, 1.1675986884,0 .,-2.5363835711| \mathrm{H}, 0.940827$ $067,0 .,-3.5917562732|\mathrm{H}, 2.1932631126,0 .,-2.2152474883| \mathrm{C}, 0.1922214972,0$. $,-0.1367265887|\mathrm{~N}, 2.7249758799,0 .,-0.1561444153| \mathrm{H}, 3.4549794302,0 ., 0.604$ 4063609|| Version=x86-Win32-G98RevA.11.4 | State=3-A' | HF=-470.3330458|RMS $\mathrm{D}=4.745 \mathrm{e}-006|\mathrm{RMSF}=1.788 \mathrm{e}-005| \mathrm{Dipole}=-0.1255509,0 ., 0.5863305 \mid \mathrm{PG}=\mathrm{CS} \quad[\mathrm{SG}($ C11H9N1) ] || e

\section{ROHF/6-311G(d)}

$1|1|$ UNPC-UNK |FOpt |ROHF | 6-311G (d) |C11H9N1 (3) |PCUSER|15-DeC-2003|0||\#P R OHF /6-311G (D) OPT SCF (CONVER=5) GEOM=CHECKPOINT GUESS=READ||molecule 4|| $0,3|\mathrm{C}, 1.4621093152,0 ., 0.5748323876| \mathrm{C}, 1.4704296028,0 ., 1.9477283399 \mid \mathrm{H}$ , $2.417874686,0 ., 2.4583480432|\mathrm{C}, 0.2871460438,0 ., 2.7040844373| \mathrm{H}, 0.334973$ $0688,0 ., 3.777758201|\mathrm{C},-0.9024619999,0 ., 2.0583533786| \mathrm{H},-1.8231246662,0$. $, 2.6140092467|C,-0.9724617784,0 ., 0.6394677933| C,-2.251847533,0 ., 0.0290$ $396604|\mathrm{H},-3.1248340986,0 ., 0.6566449464| \mathrm{C},-2.3656511394,0 .,-1.320365949$ $8|\mathrm{H},-3.3323159861,0 .,-1.7912260511| \mathrm{C},-1.2078404125,0 .,-2.112348808 \mid \mathrm{H},-$ $1.3164975889,0 .,-3.1818888795|C, 0.0649834414,0 .,-1.5841767259| C, 1.1513$ $78217,0 .,-2.5420871483|\mathrm{H}, 0.8950273747,0 .,-3.5842883515| \mathrm{H}, 2.177177405,0$ $.,-2.2652441807|\mathrm{C}, 0.2080923327,0 .,-0.1431231762| \mathrm{N}, 2.6704472782,0 .,-0.1$ $080708451|\mathrm{H}, 3.4153323197,0 ., 0.5639578077| \mid$ Version=x86-Win32-G98RevA.11 $.4 \mid$ State=3-A ${ }^{\prime}|H F=-476.2455011| \mathrm{RMSD}=7.168 \mathrm{e}-006|\mathrm{RMSF}=4.042 \mathrm{e}-005| \mathrm{Dipole}=-$ $0.1554674,0 ., 0.6787356|\mathrm{PG}=\mathrm{CS} \quad[\mathrm{SG}(\mathrm{C} 11 \mathrm{H} 9 \mathrm{~N} 1)]| \mid @$

\section{ROHF/6-311G(d,p)}

1 | 1 |UNPC-UNK |FOpt |ROHF |6-311G (d,p) |C11H9N1 (3) |PCUSER|15-Dec-2003|0||\#P ROHF / 6-311** OPT SCF (CONVER=5) GEOM=CHECKPOINT GUESS=READ||molecule 4|| $0,3|\mathrm{C}, 1.4620948858,0 ., 0.5748651849| \mathrm{C}, 1.4706096888,0 ., 1.9475857126 \mid \mathrm{H}$ $, 2.4188100004,0 ., 2.4566588801|\mathrm{C}, 0.287355492,0 ., 2.7036567749| \mathrm{H}, 0.335233$ $9711,0 ., 3.7772920466|\mathrm{C},-0.9021756261,0 ., 2.0581867751| \mathrm{H},-1.8234306037,0$ $., 2.6128470889|C,-0.9722188364,0 ., 0.6393470564| C,-2.2515839725,0 ., 0.02$ $90702976|\mathrm{H},-3.1237033396,0 ., 0.6577412762| \mathrm{C},-2.3655967906,0 .,-1.3201810$ $521|\mathrm{H},-3.3324462018,0 .,-1.7907236184| \mathrm{C},-1.2082627326,0 .,-2.1124892591 \mid$ $\mathrm{H},-1.3156474384,0 .,-3.1821952032|\mathrm{C}, 0.064520809,0 .,-1.5843836356| \mathrm{C}, 1.15$ $13786666,0 .,-2.5420407575|\mathrm{H}, 0.8919010195,0 .,-3.5838338635| \mathrm{H}, 2.17675299$ $89,0 .,-2.2603299185|\mathrm{C}, 0.2080927636,0 .,-0.1434533341| \mathrm{N}, 2.670333667,0 .,-$ $0.1079664276|\mathrm{H}, 3.4149078391,0 ., 0.5673257259| \mid$ Version=x86-Win32-G98RevA $.11 .4 \mid$ State=3-A' $|\mathrm{HF}=-476.2638172| \mathrm{RMSD}=6.248 \mathrm{e}-006|\mathrm{RMSF}=1.159 \mathrm{e}-004| \mathrm{Dipol}$ $\mathrm{e}=-0.1530886,0 ., 0.6660057|\mathrm{PG}=\mathrm{CS} \quad[\mathrm{SG}(\mathrm{C} 11 \mathrm{H} 9 \mathrm{~N} 1)]| \mid \mathrm{e}$

\section{ROB3LYP/6-311G(d,p)}

1 | 1 | UNPC-UNK | FOpt |ROB3LYP | 6-311G (d,p) |C11H9N1 (3) |PCUSER | 16-Dec-2003|0 | 
|\#P ROB3LYP/6-311** OPT SCF (CONVER=5) GEOM=CHECKPOINT GUESS=READ $\mid$ mol ecule 4||0,3|C,1.5050916283,0 . 0.5542363701|C,1.4886592267,0.,1.994440 $022|\mathrm{H}, 2.4494026443,0 ., 2.499044324| \mathrm{C}, 0.3177066,0 ., 2.7222303666 \mid \mathrm{H}, 0.3475$ $260507,0 ., 3.8060295785|\mathrm{C},-0.9000617585,0 ., 2.0581895279| \mathrm{H},-1.8270545193$ $, 0.2 .6204348565|\mathrm{C},-0.9758080855,0 ., 0.6378242208| \mathrm{C},-2.2557960995,0 ., 0$. $0284850425|\mathrm{H},-3.1340579485,0 ., 0.6636714935| \mathrm{C},-2.3802303925,0 .,-1.34949$ $63936|\mathrm{H},-3.3621799265,0 .,-1.8094104857| \mathrm{C},-1.2459764484,0 .,-2.141961469$ $6|\mathrm{H},-1.3442459713,0 .,-3.2217948805| \mathrm{C}, 0.0751166505,0 .,-1.6024558933 \mid \mathrm{C}, 1$ $.1341919279,0 .,-2.5165699864 \mid \mathrm{H}, 0.9009148976,0$, $-3.5752354879 \mid \mathrm{H}, 2.16178$ $00347,0 .,-2.1980845505|\mathrm{C}, 0.2145216499,0 \ldots,-0.1557651004| \mathrm{N}, 2.6464146799$, $0 .,-0.1036211679|\mathrm{H}, 3.4185225858,0.0 .5657530874| \mid$ Version=x86-Win32-G98 RevA.11.4|State=3-A' $|\mathrm{HF}=-479.3741446| \mathrm{RMSD}=2.840 \mathrm{e}-006|\mathrm{RMSF}=9.789 \mathrm{e}-005| \mathrm{D}$ ipole $=-0.4353652,0 ., 0.6580324|\mathrm{PG}=\mathrm{CS} \quad[\mathrm{SG}(\mathrm{C} 11 \mathrm{H} 9 \mathrm{~N} 1)]| \mid \mathrm{a}$ 